\title{
Minimum Bias Trigger Scintillators in ATLAS Run II
}

\section{A. Sidoti ${ }^{a *}$ on behalf of the ATLAS TDAQ Collaboration}

${ }^{a}$ Istituto Nazionale di Fisica Nucleare - Sezione Roma,

Piazzale A. Moro 2, Rome, Italy

E-mail: antonio.sidotieromal.infn.it

\begin{abstract}
The Minimum Bias Trigger Scintillators (MBTS) delivered the primary triggers for selecting events from real LHC collisions with the smallest bias for the low luminosity LHC RunI fills from 2009-2013 (proton-proton, lead-lead and lead-proton collisions). Similarly, for the next RunII of LHC (2015-2018) MBTS will provide key ingredients for the first physics measurements at larger LHC collisions energy (charge multiplicity, proton-proton cross section, rapidity gap measurements, ...) and in general for low luminosity LHC fills.

After more than $25 \mathrm{fb}^{-1}$ of proton-proton collisions delivered during RunI, MBTS detectors have been substantially upgraded in preparation of RunII. The upgrade strategy is presented including the scintillator replacement, the modified read out scheme, the optical measurements on RunI scintillators assessing the degradation due to the dose received.
\end{abstract}

KeYWORDS: Trigger; Minimum Bias; LHC.

${ }^{*}$ Corresponding author. 


\section{Contents}

1. Introduction [1]

2. MBTS Detector in RunI 目

2.1 Performance in RunI

3. MBTS in RunII 5

3.1 Design and Construction 5

3.2 Tests of Detectors 5

3.3 Improvements for RunII 6

田. Conclusion 6

\section{Introduction}

During the low luminosity fills of RunI (2009-2013) at the LHC, the ATLAS detector had to select online events produced by genuine collisions with the highest efficiency and with the lowest possible bias. This happened in various data taking periods: the first LHC fills at new center of mass energy, Van der Meer scans to calibrate the luminosity measurement, in the runs dedicated to the forward physics program or heavy ion collisions which include lead-lead $(\mathrm{Pb}+\mathrm{Pb})$ and protonlead $(\mathrm{p}+\mathrm{Pb})$ collisions. The Minimum Bias Trigger Scintillators (MBTS) mounted on the inner face plate of the electromagnetic endcap calorimeters, provided the most efficient and less biased trigger during low luminosity running.

Fundamental measurements such as, for example, charge multiplicity [2], underlying event characteristics [3], rapidity gap [ [4] are possible only with events collected during low luminosity fills with a small probability of pile up events. These measurements are fundamental since they characterize the Monte Carlo simulation tuning needed to get the most precise predictions for almost all ATLAS analyses. In this report we briefly recall the MBTS detector, overview its performance, describe the design foreseen for RunII and discuss the tests made so far.

\section{MBTS Detector in RunI}

The MBTS consist of $2 \mathrm{~cm}$ thick polystyrene scintillator disks mounted on both sides of the interaction point at a distance of approximately $3.6 \mathrm{~m}$ along the beam pipe. Each side has an inner and outer ring in $\eta$ of eight counters in the azimuthal angle $\phi$ (fig. (1). The outer counters pseudorapidity acceptance is $2.08 \leq|\eta| \leq 2.78$, while the acceptance for inner counters is $2.78<|\eta|<3.75$. Wavelength shifting fibers (WLS) are embedded in grooves at the edges of the counters and are grouped at the center of the module between the two pieces of scintillator. These optical fibers 


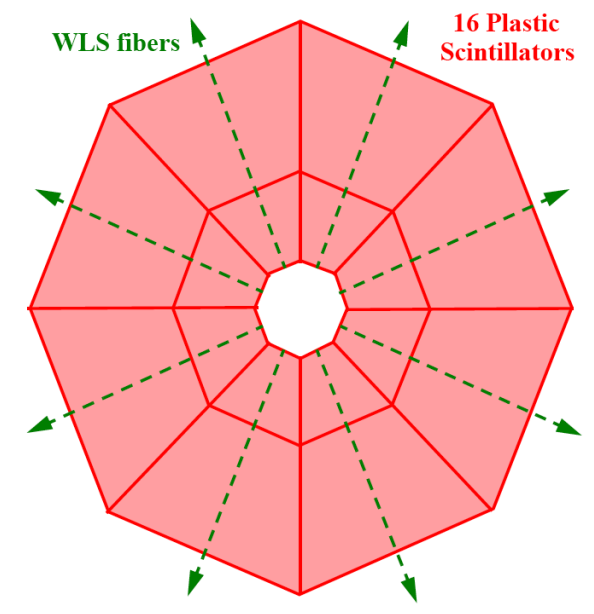

Figure 1. Layout of one of the two MBTS disks in RunI [Z]].

guide the emitted light to PMTs which are read out through the same electronics that is currently used to read out the Tile hadronic calorimeter (TileCal) [四. The MBTS signals, after being shaped and amplified ins suche a way that the pulse amplitude is proportional to the amount of energy deposited in the counter, are fed into leading edge discriminators and sent as 25 ns NIM pulses to the Central Trigger Processor (CTP) [5]. The total charge collected as well as the arrival time of the signals are recorded. An MBTS hit is defined as a signal above the discriminator threshold. At the CTP input, the 32 MBTS signals are stretched to $200 \mathrm{~ns}$ and the hit multiplicity is calculated for each side independently. The CTP combines individual signals in L1 trigger items like, for example L1_MBTS_1, L1_MBTS_2 and L1_MBTS_1_1, which require at least one, two and at least one hit per side, respectively in the MBTS detectors.

We recall that the ATLAS trigger system is composed of three levels; the first level (L1) is a hardware-based system using information from the calorimeter, muon subdetectors and forward detectors including MBTS, the second (L2) and third (Event Filter, EF ) levels are software-based systems using information from all subdetectors. Together, L2 and EF are called the High Level Trigger (HLT) [可].

\subsection{Performance in RunI}

MBTS provided fundamental information for event selections in many measurements, in particular in those releated to Soft QCD physics (e.g. [[]], [3], [四]). Performance of the MBTS subdetector has been studied for different data taking periods [ [7, []]. In fig. 目(left), the efficiency of L1_MBTS_1 trigger as a function of the offline reconstructed track multiplicity is shown. The tracks are reconstructed with tracking algorithms tuned for the low luminosity period requiring minimum track transverse momentum, $P_{T}>100 \mathrm{MeV} / c$, and absolute pseudorapidity $|\eta| \leq 2.5$ ). The efficiency is larger than $95 \%$ even for very small track multiplicities. Fig. 目 (right) shows the efficiency as a function of the azimuthal angle $\phi$ is shown with tracks reconstructed by the ATLAS Inner Detector (ID) and intersecting the MBTS detectors. Only a small $\eta$ coverage of the MBTS detector which overlaps with the ID coverage is probed. However a reasonable agreement with simulation is observed with the exception of the edge of the detectors. 

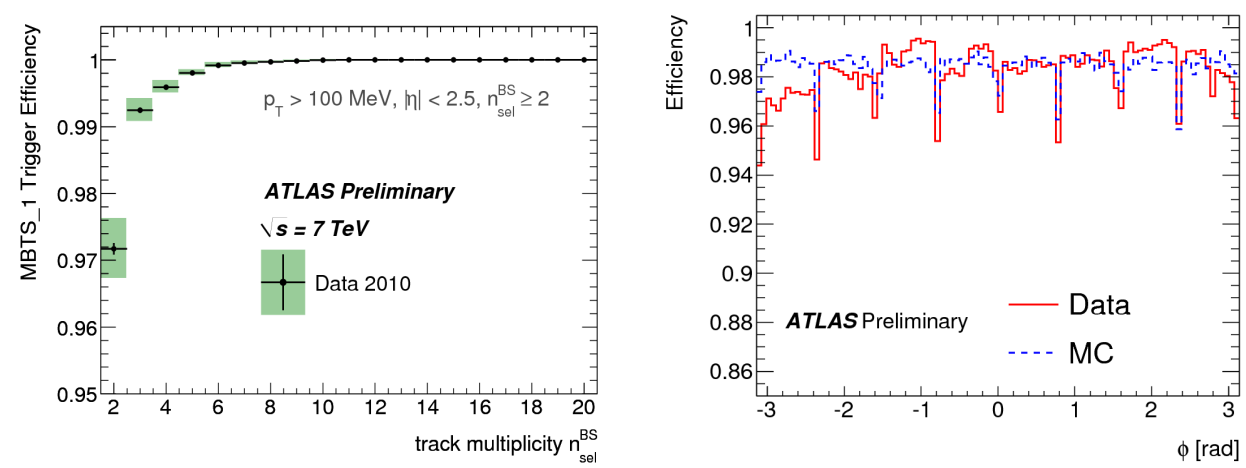

Figure 2. Efficiency of the L1_MBTS_1 trigger as a function of the offline track multiplicity (left), and azimuthal angle $(\phi)$ for single tracks reconstructed in the ID crossing the MBTS module (right) [ 8$]$ ].

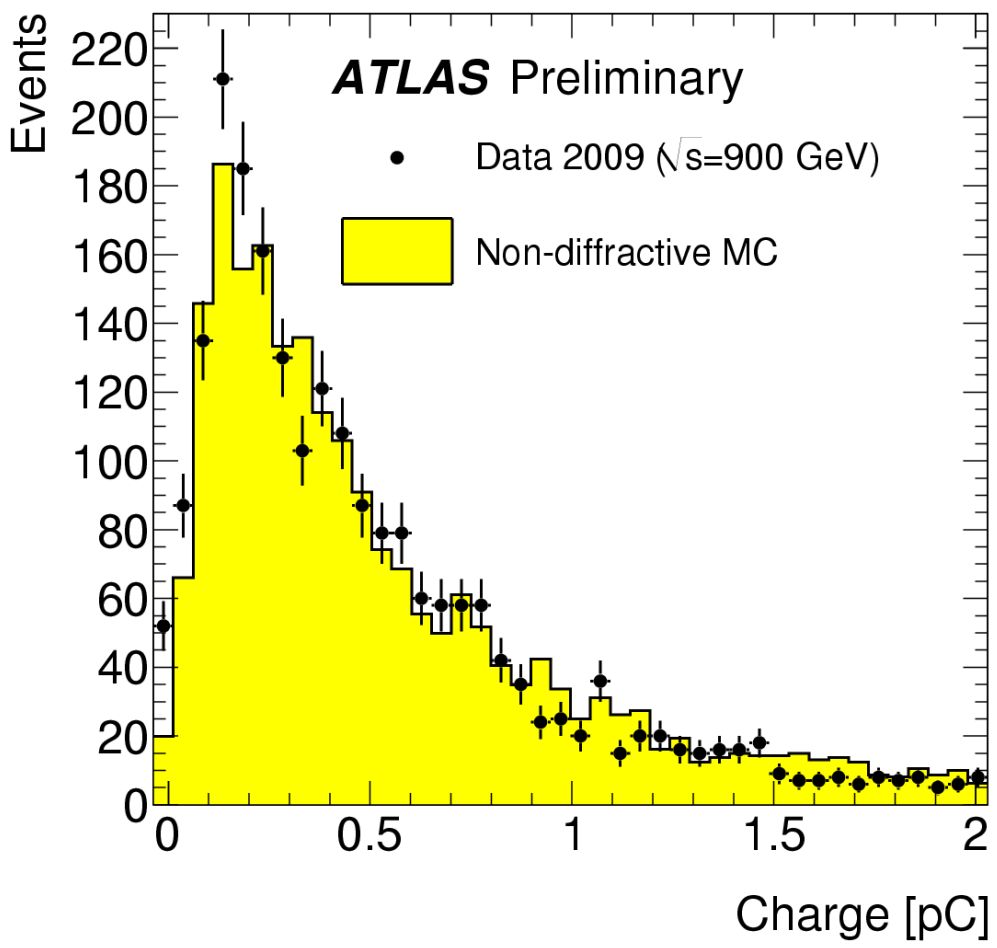

Figure 3. Charge spectrum for a single MBTS counter for events with exactly one track is extrapolated from the ID to the MBTS module [Z].

The simulation has been tuned such to have an excellent agreement of the charge collected by the PMTs with data which is shown in fig. 3.

The performance shown so far was relative to 2010 LHC fills ( $\mathrm{p}+\mathrm{p}$ collisions at $\sqrt{s}=900 \mathrm{GeV}$ and $\sqrt{s}=7 \mathrm{TeV}$ ). Although the MBTS was not expected to be operated after receiving a large radiation dose ${ }^{1}$, it was used successfully to trigger on later $\mathrm{LHC}$ fills, in particular in $\mathrm{Pb}+\mathrm{Pb}$ collisions

\footnotetext{
${ }^{1}$ The dose received varies with the radial distance from the beam line and has been estimated to be in the range of $0.1 \sim 0.4 \times 10^{4}$ Gy
} 

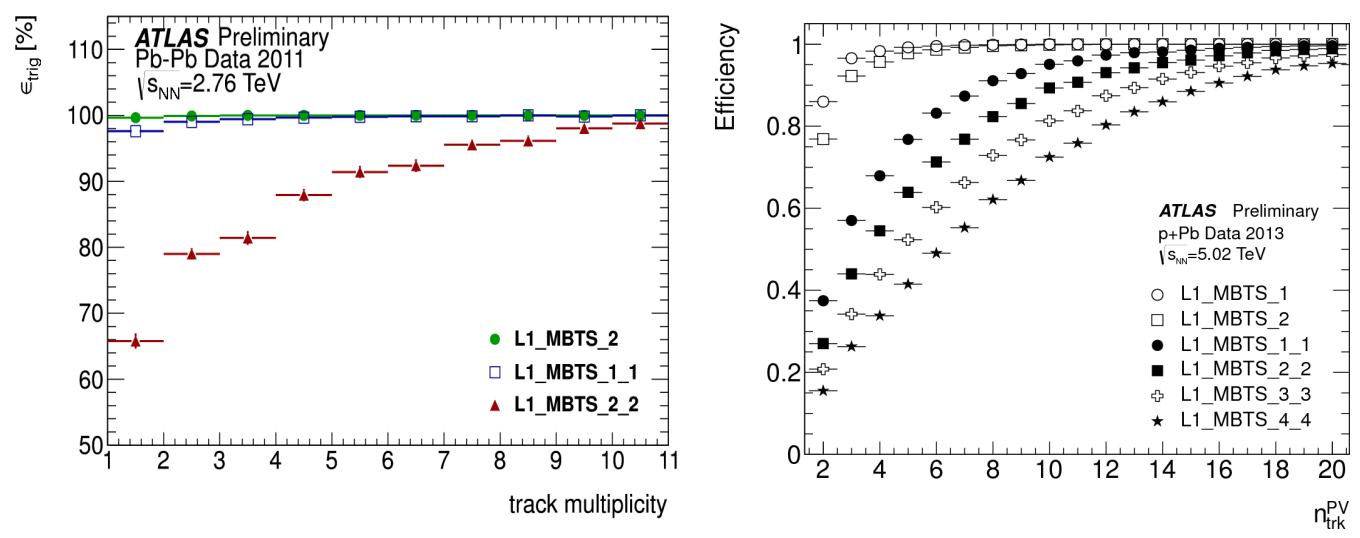

Figure 4. MBTS trigger efficiency as a function of the number of reconstructed tracks in $2011 \mathrm{~Pb}+\mathrm{Pb}$ data taking (left) [9], and in 2013 p+Pb runs (right) [10]. L1_MBTS_X defines a trigger where the sum of counters above threshold on any side is at least X. L1_MBTS_Y_Y defines a trigger where at least Y counters on both sides (AND) are above threshold.
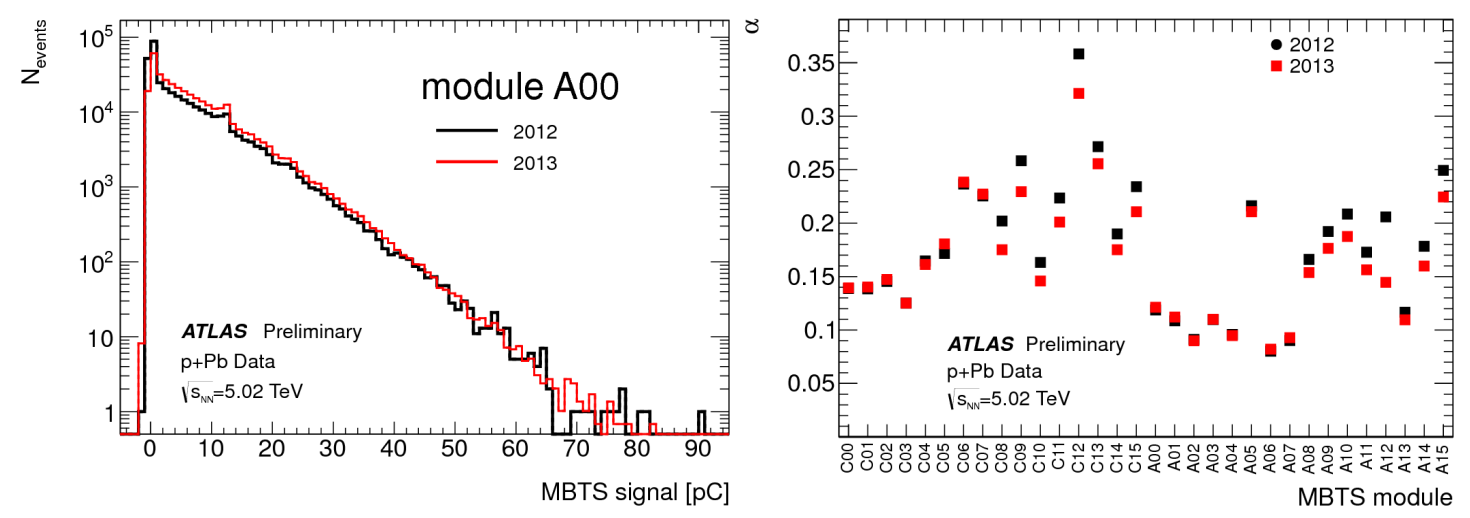

Figure 5. (Left) Example of typical signals collected by an MBTS module during two RunI p+Pb data taking periods: Sept. 2012 and Jan. Feb. 2013. (Right) The slope parameter from the exponential parameterization $e^{-\alpha x}$ of the MBTS signal distribution for all MBTS modules for events accepted by an orthogonal trigger obtained in p+Pb collisions in the years 2012 (black) and 2013 (red). For each side (A or C) counters with index 00-07 and 08-15 are respectively located in the outer and inner $\eta$ ring [10]

in 2011, the first p+Pb LHC collisions in Sept. 2012 and the longer 2013 p+Pb LHC runs (Jan. 2013-Mar. 2013). Fig. $\$$ (left) shows the MBTS trigger efficiency of the most important trigger chains using MBTS as a function of the number of offline reconstructed tracks in the $2011 \mathrm{~Pb}+\mathrm{Pb}$ data taking. Fig. $\$$ (right) shows similar efficiency for the 2013 p+Pb data taking. It is interesting to note that $\sim 20 \mathrm{fb}^{-1}$ of integrated luminosity from $\mathrm{p}+\mathrm{p}$ collisions has been delivered to ATLAS between these two results. Also, because of the different kind of collisions $(\mathrm{Pb}+\mathrm{Pb} \quad$ vs $\mathrm{p}+\mathrm{p})$ and different high voltages PMT settings, the two data taking periods are not easily comparable. The MBTS based triggers provided still large efficiency even for very low track multiplicity events.

A comparison of the signal loss between two identical $\mathrm{p}+\mathrm{Pb}$ data taking periods, the first in Sept. 2012 the other one in Jan. 2013 after $\sim 5 \mathrm{fb}^{-1}$ of integrated luminosity from $\mathrm{p}+\mathrm{p}$ collisions between the two has been performed by plotting the exponential slope of the charge collected by 
the different MBTS detectors (fig. 5 (left)). Fig. 5 (right) shows the exponential slope parameter measured in 2012 and 2013 for each MBTS counter. As expected, light yields for the inner ring counters seems to be more affected than the outer rings.

\section{MBTS in RunII}

\subsection{Design and Construction}

It is estimated that in RunI, MBTS received a dose ${ }^{2}$ in the range of $0.1 \sim 0.4 \times 10^{4}$ Gy. Since in Run II, the same Soft QCD measurements have to be performed for the new LHC energy $\sqrt{s}=13$ $\mathrm{TeV}$, it was decided to replace the MBTS with a new detector based on the same ideas and trying to keep the same materials. However, the number of read out channels has to be reduced since they will be in use by the TileCal to instrument the "gap regions" (kept uninstrumented during RunI). The readout scheme was partially modified for RunII: the outer ring detectors light connectors have been merged two by two, while the inner rings have been kept as they were in RunI. The WLS fibers path has been modified and run closer to the detector edges, reducing the dips in the $\phi$ efficiency observed in RunI (fig. 目 (right)). The $\eta$ coverage has been slightly modified,with the outer and inner ring covering respectively $2.08 \leq|\eta|<2.76$ and $2.76 \leq|\eta| \leq 3.86$. The various operations of cutting, machining, assembly and wrapping of the scintillators have been performed in Winter 2014. The side A of MBTS detectors was installed in February 2014, while C side will be installed in July 2014.

\subsection{Tests of Detectors}

Various tests have been performed on the new scintillators as well as the old ones extracted from the detectors used in RunI (i.e. after irradiation).

Relative light transmission has been checked using a movable $\mathrm{Sr}$ source on a motorized table. Results are shown in fig. 6 (left). Note that a direct comparison between the two types of scintillators is not possible since the $\mathrm{Sr}$ scans tests have not been intercalibrated passing from one sample to the other ${ }^{3}$. However the reduction in the light transmissions caused by the WLS fiber is clearly visible in the RunI sample with respect to the new WLS path for Run II. There is also only a moderate dependence in the light transmission in the vertical dimension ( $r$ axis) showing that the damage from radiation, which is strongly dependent on $r$ coordinate, seems under control.

After assembly each module has been tested with the so called Cs scans used to check the ATLAS TileCal [11]. Each MBTS detector has been placed on one side of the ATLAS TileCal module while a $C s$ source circulated in the water lines inside the module at different radial distances. When the source passes in the proximity of the scintillator, the two PMTs (one for the inner and one for the outer sub-module) collect some light from the scintillators. Typical test results are shown in fig. (right). These tests have been used to cross check the optics quality and the assembly of each MBTS detector before installation.

\footnotetext{
${ }^{2}$ Roughly, a loss of $50 \%$ of light transmission is expected after receiving a dose of $10^{4} \mathrm{~Gy}$.

${ }^{3}$ In particular the activity of the Sr source and the speed of the movable source is not constant between one scan and the other, and due to the size of the table, it was not possibile to measure in the same time the two counters.
} 

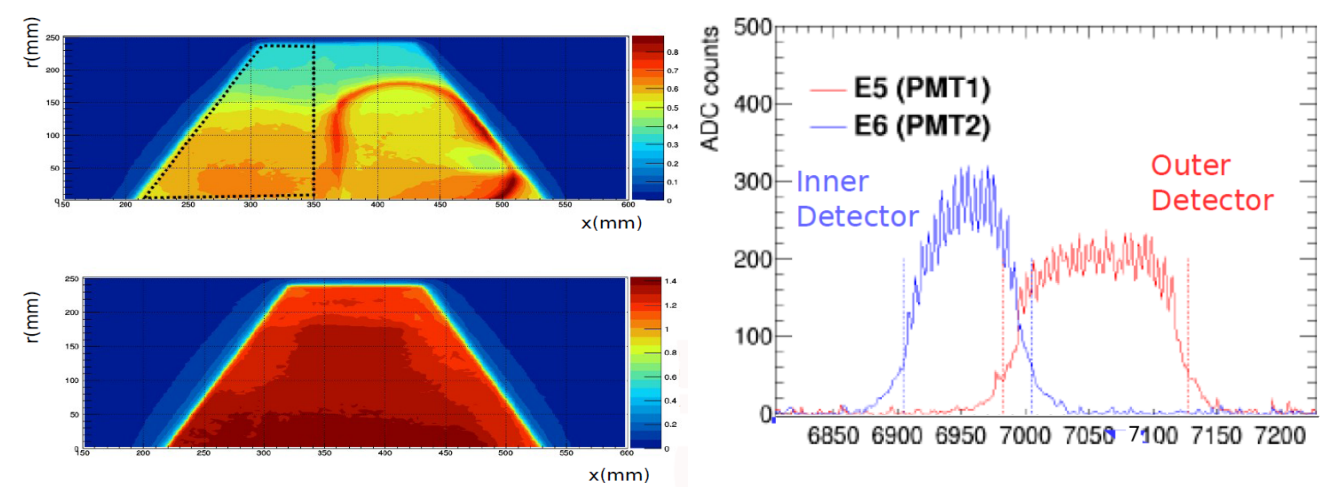

Figure 6. (Left) Relative light transmission maps obtained with $\mathrm{Sr}$ scans for inner MBTS detectors RunI irradiated (top) and the new one built for RunII (bottom). A direct comparison for both samples is not possible since there is no intercalibration between the two measurements. (Right) Cs scan result. The dotted region on the top left indicates the half MBTS counter is upside down with respect to the top right. More precisely, the groove hosting the WLS fiber is the same (opposite) side with respect to the $S r$ source for the top right (top left) portion of the plot. The charge collected by the PMT output is shown as a function of the position in $\mathrm{cm}$ (the origin is arbitrary) of the $C s$ source along the water lines inside the tile. The fast modulation of the signal is caused by the Cs decay products crossing alternatively the iron (absorber) and the tile (scintillator) parts of the TileCal module, while the "slow" modulation is caused by the Cs source approaching the region where the MBTS detector is placed.

From the improved RunII design and the tests performed we foresee to have, approximately, the same light yield of the outer MBTS modules, and to double it for the inner MBTS modules with respect to the RunI setup.

\subsection{Improvements for RunII}

Some improvements have been foreseen to fix few issues observed during RunI data taking.

Firstly signal reflections were observed between different components of the DAQ chain in particular between those placed in the ATLAS Cavern and the ones located in the underground counting room, located $80 \mathrm{~m}$ away, causing a non negligible accidental fake rate. The impedance mismatch of all the adapter boards has been fixed.

Instead of using leading edge discriminators, in Run II, constant fraction discriminators will be used to avoid excessive timewalk caused by the large dynamical range of the signals coming from the MBTS modules (fig. 5 (left)).

\section{Conclusion}

The MBTS system will play a fundamental role in the ATLAS experiment during low luminosity LHC fills in the RunII data taking. During the 2013-2014 shutdown period, MBTS has been upgraded. The commissioning phase of the MBTS detector will start in the second half of 2014 and continue until the start up of RunII with the determination of the optimal operating points. 


\section{Acknowledgments}

It is a pleasure to thank Ana Henriques, Sabrina Sacerdoti, Oleg Solovyanov, Giulio Usai for useful discussions.

\section{References}

[1] Atlas Collaboration, Readiness of the ATLAS Tile Calorimeter for LHC collisions Eur.Phys. J. C 70 (2010) 1193.

[2] ATLAS Collaboration, Measurement of the inelastic proton-proton cross-section at $\sqrt{s}=7$ TeV with the ATLAS detector, Nature Communications 2 (2011) 463.

[3] ATLAS Collaboration, Measurement of underlying event characteristics using charged particles in pp collisions at $\sqrt{s}=900 \mathrm{GeV}$ and $7 \mathrm{TeV}$ with the ATLAS detector, Phys. Rev. D 83 (2011), 112001.

[4] ATLAS Collaboration, Rapidity gap cross sections measured with the ATLAS detector in pp collisions at $\sqrt{s}=7$ TeV, Eur. .Phys. J. C72 (2012) 1926, arXiv1201:2808.

[5] S. Ask and others, The ATLAS central level-1 trigger logic and TTC system, JINST 3 (2008), P08002.

[6] ATLAS Collaboration, Performance of the ATLAS Trigger System in 2010, Eur.Phys.J. C72 (2012), 1849.

[7] ATLAS Collaboration,Performance of the Minimum Bias Trigger in p-p Collisions at $\sqrt{s}=900 \mathrm{GeV}$ ATLAS-CONF-2010-025, http://cds.cern.ch/record/1277657.

[8] ATLAS Collaboration, Performance of the Minimum Bias Trigger in p-p Collisions at $\sqrt{s}=7 \mathrm{TeV}$ ATLAS-CONF-2010-068, http://cds.cern.ch/record/1281343.

[9] ATLAS Collaboration, Performance of the ATLAS Minimum Bias and Forward Detector Triggers in 2011 Heavy Ion runs A TLAS-CONF-2012-122, http: / / cds . cern. ch/record/1473425

[10] ATLAS Collaboration, Performance of the ATLAS Minimum Bias and Forward Detector Triggers in pA collisions, ATLAS-CONF-2013-104, http: / / cdsweb. cern. ch/record/1624013

[11] O. Solovyanov for the ATLAS Collaboration Performance of the ATLAS Tile Hadronic Calorimeter at LHC in Run 1 and planned upgrades, these proceedings. 